\title{
PCR Amplification of a Superoxide Dismutase Gene (pasod) from Psychrophilic Bacteria (KOPRI22215) without Genome Information
}

\author{
Jumee Na and Kyunghee Lee* \\ Department of Chemistry, Sejong University, Seoul 143-747, Korea. ${ }^{*}$ E-mail: khlee@sejong.ac.kr \\ Received June 15, 2010, Accepted June 29, 2010
}

Key Words: Superoxide dismutase, Cold-adapted, PCR

Aerobic organisms have adopted delicate defense mechanisms using some antioxidant enzymes, such as superoxide dismutase (SOD) and catalase (CAT) in order to protect themselves from oxidative stress. In most cases, oxidative stresses are caused by a number of reactive oxygen species (ROS) generated as byproducts of respiration. ${ }^{1}$ SOD is a ubiquitous metalloenzyme, detoxifying ROS by catalyzing the dismutation of toxic superoxide anions into $\mathrm{O}_{2}$ and $\mathrm{H}_{2} \mathrm{O}_{2}$. In bacteria, four different SODs (Fe-SOD, Mn-SOD, CuZn-SOD, and Ni-SOD) exist with characteristic structural features. ${ }^{2}$ For example, SODs from many extremophiles belong to a family of Fe/Mn-SODs, which are composed of an $\mathrm{N}$-terminal domain with a long alpha antiparallel hairpin and a $\mathrm{C}$-terminal domain with three-stranded antiparallel $\beta$-sheets, flanked on both sides by four-a helices according to Pfam hits. ${ }^{3}$ Depending on the type of metals, they also have different properties in terms of their oligomeric states as well as selective inhibition. In general, the Fe-SODs form homodimers whereas the Mn- SODs form homotetramers in solution. FeSOD is inactivated by hydrogen peroxide whereas MnSOD is inactivated by cyanide.

In this study we attempted to identify a sod gene from psychrophilic bacteria which grow in extremely cold region such as the Arctic. Molecular mechanisms by which psychophilic bacteria have developed cold-resistance still lacks confirmation even though some comparison studies between psychrophilic and mesophilic bacteria have been reported. ${ }^{4}$ Psychrophilic bacteria provide a good target group since they have adapted themselves to survive in a permanently cold environment, such as the polar region where the oxygen concentration is rather high. So far there are only three psychrophilic SODs reported: Marinomonas sp NJ522, Pseudoalteromonas haloplanktis, and Aliivibrio salmonicida. ${ }^{5}$ In P. haloplanktis, PhSOD is a homodieric Fe-containing enzyme and is active even at a low temperature. The highly reactive Cys57 residue leads to the formation of a disulfide bridge between two monomeric subunits. ${ }^{6}$ Glutathionylation of SOD was later characterized and it was suggested that it might provide a cold-adaptation strategy. ${ }^{7}$ Recently, the structure of a cold-adapted SOD was demonstrated with $A$. salmonicida, whose FeSOD exhibits lower thermostability compared with that of Escherichi coli (E. coli) probably due to its surface having an increased net negative charge. ${ }^{8}$

It is of interest to us to search for cold-adapted SODs from Psychromonas arctica (KOPRI22215) ${ }^{9}$ and compare them with those of $E$. coli. It is accordingly inferred that $P$. arctica might have developed an efficient protection system against oxidative stress resulting from reduced metabolic turnovers in respiration pathways. With the aim of identifying a coding gene for SOD from $P$. arctica, we have performed a series of polymerase chain reactions (PCR) (Fig. 1A). Since there is no genomic information available for $P$. arctica so far we designed a set of primers by aligning the encoding sod genes from Psychromonas ingrahamii, Shewanella frigidimara, and Polaribacter irgensii using ClustalW2 (EBI server). ${ }^{10}$ The two consensus sequences were targeted at the 5'-ATG containing region ( $\mathrm{M}_{1}$ A F E L P A L P $\left.\mathrm{Y}_{10}\right)$ and the 3'-near region $\left(\mathrm{T}_{156} \mathrm{~V}\right.$ D V W E H A Y Y $\left.{ }_{165}\right)$, which were probed as two primers [ 5'-ATG GCT TTT GAA TTA CCA GCA TTA CCC TAT G-3' (forwarding primer; F1) and 5'-GT AGT ATG CAT GTT CCC ATA CAT CTA CAG TT-3' (reversing primer; R1)]. Gradient PCR was performed with F1 and R1 primers to yield a DNA fragment of a 430 base pair (data not shown). Annealing with two primers was carried out at $51.2{ }^{\circ} \mathrm{C}$ for $30 \mathrm{sec}$ and extension was performed at $72{ }^{\circ} \mathrm{C}$ for $2 \mathrm{~min}$. After 20 cycles, the PCR products were analyzed in a $1.0 \%$ agarose gel and stained with ethidium bromide $(\mathrm{EtBr})$. Amplified DNA was extracted from the single band corresponding to the desired molecular weight ( $\sim 410 \mathrm{bp})$ and sequenced to provide the nucleotide sequence of the amplified fragment. In order to discover the remaining flanking sequences of the newly identified $410 \mathrm{bp}$ fragment, LA (long \& accurate) in vitro PCR technology (Takara) was employed. ${ }^{11}$ In the LA PCR me-

(A)

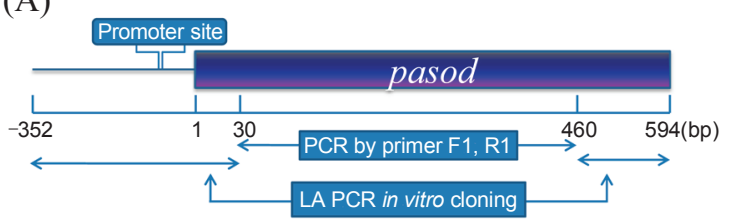

(B)

ATGAATTTGCGATTATTC TATCATAGACACAAAATGAATCTAGATCA ATCTTTATGGTGGCTTTTTATTCTTAAAGTAAGTATAAATAACCACT ATAAATTTAGATCCCCCCCCAGCCTTTATTAGGAGAATTTAAAATG

; promoter, $\square$; Pribnow box, +1 ; transcription start, $A T G$; translation start

Figure 1. The identified pasod gene from $\boldsymbol{P}$. arctica. (A) The pasod gene was amplified from genomic DNA by a series of homologous PCRs and LA in vitro PCR technology. The full length of the pasod gene (594bp) was identified by sequencing of the amplified product along with upstream promoter sequences. (B) The promoter region of the pasod gene was identified by Neural Network Promoter prediction. 
thod any unknown region of the genome can be amplified using cassettes containing specific restriction enzyme sites and their cognate primers. In this study, genomic DNA was digested with Pst1 and ligated with Pst1 cassettes. The 1st PCR was carried out with a cassette primer $(\mathrm{C} 1)$ and a specific primer $(\mathrm{F} 1)[\mathrm{C} 1$ : 5'-GTA CAT ATT GTC GTT AGA ACG CGT AAT ACG ACT CA-3', F1(used for the 1st gradient PCR): 5'- ATG GCT TTT GAA TTA CCA GCA TTA CCC TAT G -3']. Annealing was done at $55{ }^{\circ} \mathrm{C}$ for $30 \mathrm{sec}$ and extension was performed at $72{ }^{\circ} \mathrm{C}$ for 1 min using Takara LA Taq polymerase. The subsequent 2nd PCR was carried out with $\mathrm{C} 2$ [5'-CGT TAG AAC GCG TAA TAC GAC TCA CTA TAG GGA GA-3'] and F2 (SF3) [5'-CTG GTG GCG AAC CTT CTG GTG AAC TTG CTG-3']. The resulting amplified band was sequenced to provide the complete sequence of the 3'-end region. Similarly, in order to fill in the 5'-coding region, DNA ligated with Hind III-cassettes was amplified with $\mathrm{C} 1$ and R1 (used for the 1st gradient PCR), C2 and R2 (SR5) [5'-CATGATGTTT GCCGTGGTGA AAT TCTAATG -3'] for the 2nd PCR. The completed open reading frame of the pasod gene was finally discovered as $594 \mathrm{bp}$ long. Nucleotide sequence data for the pasod gene is now available in the GenBank database under the accession number, GQ120613. Additional information of the upstream region was also discovered up to the -352 site and the promoter sequence in the upstream of ATG codon was analyzed by Neural Network Promoter prediction (UC Berkeley) as shown in Fig. 1B. ${ }^{12}$

The nucleotide sequence of pasod gene was translated to the amino acid sequence by Translate program (ExPASy Proteomic Server), which was in turn compared with other SOD proteins to search for the conserved domain using the Position-Specific Iterated BLAST (PSI-BLAST). The translated PaSOSD protein was 197 amino acid long with an Accession number of ACR83609 in the GenBank database. Alignments of PaSOD with EcSODs of mesophilic E. coli were carried out using ClustalW2 to give a score of 76 with Fe type-EcSOD and 42 with Mn type-EcSOD, suggesting that PaSOD may function as a Fe-SOD (data not shown). The secondary structure of PaSOD analyzed by PredictProtein (Columbia University) reveals that PaSOD is composed of $48 \%$ helices, $9 \%$ extended and $43 \%$ unstructured regions.

We have also attempted to express the recombinant PaSOD protein using E. coli expression systems. The pasod gene was amplified to full length using corresponding primers designed to contain Nde1 and BamH1 sites at each end [forwarding F3; 5'-CAT ATG GCT TTT GAA TTA CCA GCA TTA CC-3', reversing R3; 5'- GGA TCC CTA TAG GGA GAC TGC AGC AAA-3']. Gradient PCR was carried out at $48-58{ }^{\circ} \mathrm{C}$ to yield a maximum amplification at $54.4{ }^{\circ} \mathrm{C}$. The amplified PCR product was then purified from agarose gel and cloned into a pBluescriptII vector. The pasod gene was digested with NdeI and BamH1 and subsequently ligated into a pAED4 vector under the control of the T7 promoter system, ${ }^{5}$ previously cut with the same enzymes to provide PaSOD/pAED4. BL21(DE3) was transformed with PaSOD/pAED4 and cultured at $30{ }^{\circ} \mathrm{C}$ in $\mathrm{LB}^{\mathrm{Amp}}$ until the cell density reached O.D. $600 \mathrm{~nm}$ of 0.3, at which point IPTG (isopropyl $\beta$-D-1-thiogalactopyranoside) was added at a final concentration of $0.01 \mathrm{mM}$. After $5 \mathrm{hr}$ induction with IPTG, cells were harvested and frozen at $-70{ }^{\circ} \mathrm{C}$ and lysed on

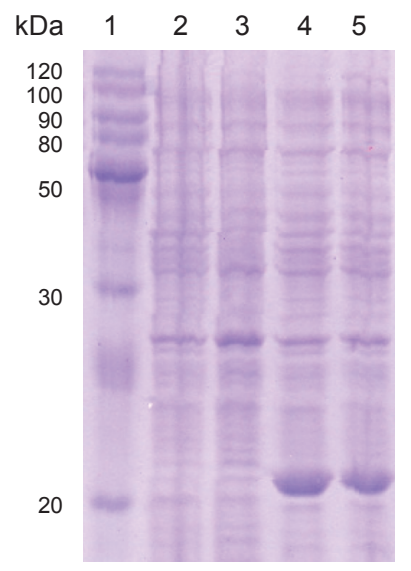

Figure 2. Expression of PaSOD in a pAED4 vector. The pasod gene was cloned into pAED4 (lane 4, 5) along with vector controls (lane 2, 3). BL21(DE3) transformants with resulting plasmids were incubated without (lane 2,4) or with $0.01 \mathrm{mM}$ IPTG (lane 3,5). Protein size markers are shown in lane 1.

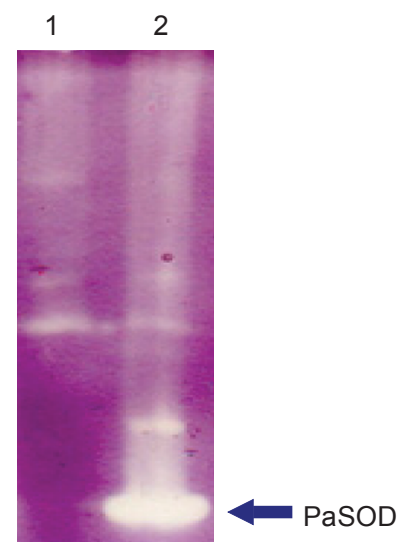

Figure 3. SOD staining in a native PAGE gel. PaSOD protein (lane 2) was separated in a native PAGE gel along with the pAED4 vector control (lane 1). The native PAGE gel was stained with NBT and riboflavin before illumination with a fluorescent lamp.

ice. The concentration of crude extract protein was measured by absorbance at $595 \mathrm{~nm}$ according to the Bradford method with bovine serum albumin as a standard. ${ }^{14}$ Fig. 2 shows that the construct successfully expressed PaSOD protein with autoinduction (lane 4) as well as $0.01 \mathrm{mM}$ IPTG induction (lane 5).

In order to check that the expressed PaSOD proteins were functional, we have performed SOD staining on native PAGE gels. ${ }^{15}$ Upon incubation in the presence of nitro blue tetrazolium (NBT) and rivoflavin solution in the dark followed by illumination with a fluorescent lamp the native gel revealed a strong white band corresponding to PaSOD protein (lane 2 in Fig. 3). As a comparison no white band was detected in the pAED4 vector control (lane 1). This finding indicates that the recombinant PaSOD folded correctly to provide the SOD activity in the native gel.

Taking all the results together, we have confirmed that we were able to identify a pasod gene from cold-adapted bacteria even in the absence of genomic information in our hands. The pasod gene was amplified to full length and further cloned 
into a pAED4-based protein expression system. Recombinant PaSOD protein was then successfully induced by IPTG and functionally active based on the native gel system. Ongoing studies on in vitro biochemical analysis with the purified PaSOD protein will give the more detailed information necessary to evaluate the activity of PaSOD as a functional candidate to provide cold adaptation in $P$. arctica. Development of His-tagged PaSOD expression system is in progress.

\section{Experimental Section}

PCR amplifications. Genomic DNA was extracted from KOPRI22215 culture using the bacteria genomic DNA extraction kit (iNtRON). Gradient PCR was performed in a 96 Gradient Thermal Block (Bioneer). The PCR mixture consisted of genomic DNA, dNTPs, each primer, $10 \times$ Taq buffer and Taq polymerase in a final volume of $20 \mu \mathrm{L}$. Annealing with primers was done at $54.4{ }^{\circ} \mathrm{C}$ for $30 \mathrm{sec}$ and extension was carried out at $72{ }^{\circ} \mathrm{C}$ for $2 \mathrm{~min}$. After 30 cycles, the PCR products were analyzed in a $1.0 \%$ agarose gel, stained with ethidium bromide (EtBr). The single band corresponding to the desired molecular weight was cut, from which the amplified DNA was purified using a Geneclean turbo kit (MP Bio). The nucleotide sequence was determined by Cosmo GenTech (Seoul, Korea).

Identification of SOD activity on native PAGE gels. Native gels without SDS and $\beta$-mercaptoethanol were prepared with $7.5 \%$ polyacrylamide. After suspending the protein crude extract in dissolving buffer (62 mM Tris- $\mathrm{HCl}(\mathrm{pH} 6.8), 0.01 \%$ bromophenol blue, $10 \%$ glycerol), $4 \mu \mathrm{g}-0.06254 \mu \mathrm{g}$ of protein sample was loaded in the native gel with dilution of 2 . The native gel was run at $80 \mathrm{~V}$ for $15 \mathrm{~min}$ and $150 \mathrm{~V}$ for $60 \mathrm{~min}$ at $4{ }^{\circ} \mathrm{C}$. The native gels were incubated in $1.23 \mathrm{mM}$ nitro blue tetrazolium (NBT) for $15 \mathrm{~min}$ at $30{ }^{\circ} \mathrm{C}$ followed by an immersion in 0.028
$\mathrm{mM}$ rivoflavin solution (100 mM phosphate buffer $(\mathrm{pH} 7.0)$ ) containing $28 \mathrm{mM}$ tetramethylethylenediamine (TEMED) with gentle shaking at $75 \mathrm{rpm}$ in the dark. After briefly washing, the gels were illuminated with fluorescent lamp (45 W) for $15 \mathrm{~min}$ to make the SOD activity visible.

Acknowledgments. This work was supported by the faculty research fund of Sejong University in 2008.

\section{References}

1. Bannister, J. V.; Bannister, W. H.; Rotilio, G. CRC Crit. Rev. Biochem. 1987, 22, 111.

2. Tainer, J. A.; Getzoff, E. D.; Beem, K. M.; Richardson, J. S.; Richardson, D. C. J. Mol. Biol. 1982, 160, 181.

3. Knapp, S.; Kardinahl, S.; Hellgren, N.; Tibbelin, G.; Schafer, G.; Ladenstein, R. J. Mol. Biol. 1999, 285, 689.

4. Feller, G.; Gerday, C. Cell Mol. Life Sci. 1997, 53, 830.

5. Zheng, Z.; Jiang, Y. H.; Miao, J. L.; Wang, Q. F.; Zhang, B. T.; Li, G. Y. Biotechnol. Lett. 2006, 28, 85.

6. Castellano, I.; Di Maro, A.; Ruocco, M. R.; Chambery, A.; Parente, A.; Di Martino, M. T.; Parlato, G.; Masullo, M.; De Vendittis, E. Biochimie. 2006, 88, 1377.

7. Castellano, I.; Ruocco, M. R.; Cecere, F.; Di Maro, A.; Chambery, A.; Michniewicz, A.; Parlato, G.; Masullo, M.; De Vendittis, E. Biochim Biophys Acta 2008, 1784, 816.

8. Pedersen, H. L.; Willassen, N. P.; Leiros, I. Acta Crystallogr. Sect. F. Struct. Biol. Cryst. Commun. 2009, 65, 84.

9. Lee, Y. K.; Jung, H. J.; Lee, H. K. J. Microbiol. 2006, 44, 694.

10. Thompson, J. D.; Higgins, D. G.; Gibson T. J. Nucleic Acids Res. 1994, 22, 4673.

11. Choi, A.; Na, J. M.; Sung, M. S.; Im, H. N.; Lee, K. Bull. Korean Chem. Soc. 2010, 31, 887

12. Pollastri, G.; Przybylski, D.; Rost, B.; Baldi, P. Proteins 2002, 47, 228.

13. Jin, J. P. J. Biol. Chem. 1995, 270, 6908.

14. Bradford, M. M. Anal. Biochem. 1976, 72, 248.

15. Beauchamp, C.; Fridovich, I. Anal Biochem 1971, 44, 276. 